\title{
Determination of finish-cutting operation number and machining-parameters setting in wire electrical discharge machining
}

\author{
J.T. Huang, Y.S. Liao *, W.J. Hsue \\ Department of Mechanical Engineering, National Taiwan University, 1, Sec. 4, Roosevelt Road, Taipei 10764, Taiwan
}

Received 9 August 1997

\begin{abstract}
Wire electrical discharge machining (WEDM) plays an important role in precision manufacturing. To obtain a precise workpiece with good surface quality, some extra repetitive finish cuts along the rough cutting contour are necessary. An attempt has been made to unveil the influence of the machining parameter (pulse-on time, pulse-off time, table feed-rate, flushing pressure, distance between wire periphery and workpiece surface, and machining history) on the machining performance of WEDM in finish cutting operations. The gap width, the surface roughness and the white layer depth of the machined workpiece surface are measured and evaluated. Based on the Taguchi quality design method and numerical analysis, it is found that the pulse-on time and the distance between the wire periphery and the workpiece surface are two significant factors affecting the machining performance. Mathematical models relating machining parameters and performance are established by regression, and non-linear programming using the Feasible-direction algorithm was used to obtain the optimal machining parameters. A strategy of optimal multi-cut WEDM process planning from rough to finish cutting operations, including the number of machining operations and their corresponding machining-parameters setting for each operation, has been proposed. Experimental results show that the proposed approach can achieve better performance than that achieved by a well-skilled operator. A better surface quality and accurate dimension value can be obtained in less machining time. (C) 1999 Published by Elsevier Science S.A. All rights reserved.
\end{abstract}

Keywords: Wire electrical discharge machining; Finish cutting; Machining parameters; Offset; Taguchi method

\section{Introduction}

The technologies of wire electrical discharge machining (WEDM) have been emphasized significantly and have improved rapidly in recent years owing to the requirements in various manufacturing fields, especially in the precision die industry. Good rigidity and the dynamic characteristic of the machine are pre-requisites to achieving optimal machining performance. In addition, proper machining-parameters setting of WEDM also plays an important role. The setting of machining parameters relies strongly on the experience of operators and machining-parameters tables provided by machinetool builders. It is difficult to utilize the optimal functions of a machine owing to their being too many adjustable machining parameters.

\footnotetext{
* Corresponding author. Fax: +886 22 3631755; e-mail: liaoys@ccms.ntu.edu.tw
}

Usually some extra repetitive finish cuts along the contour of a previous cut are necessary, by offsetting the wire by a value as shown in Fig. 1, so that the specified accuracy and good surface quality can be obtained. The finish-cutting process is a process of one-side cutting with a smaller offset value, which needs smaller electrical discharge energy and results in a faster table feed-rate. Besides the generally-required machining conditions in rough cutting, the number of finish cuts and their corresponding offset value in each cut should be specified before the start of machining operations [1]. The offset is important in process planning and its value is always given in WEDM machining-parameters setting tables. Inappropriate offset value leads to unsatisfactory accuracy, and will reduce the surface quality of the machined part also. Because of the non-contact characteristic of electrical discharge and gap-width estimation of multi-cuts, determining an optimal offset value in advance is difficult and still a challenge. 


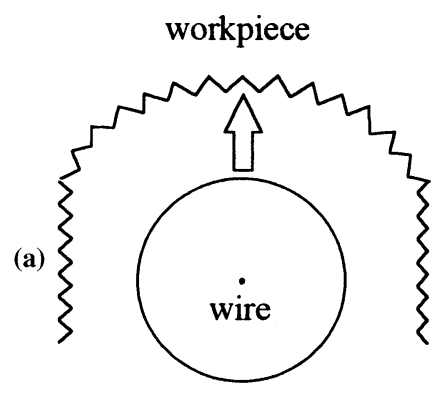

\section{Rough cutting}

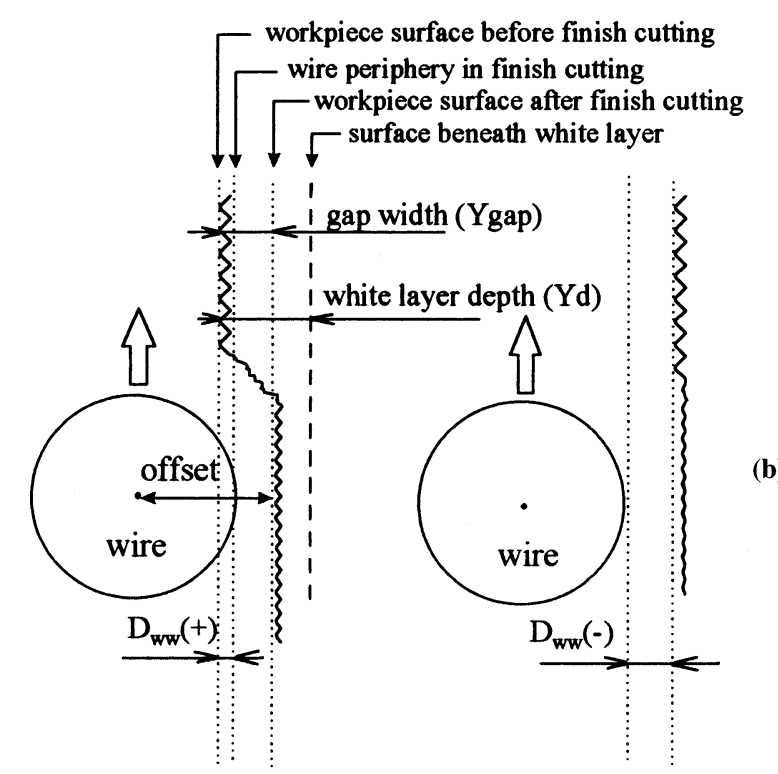

Finish cutting

Fig. 1. A schematic plan view of: (a) rough and; (b) finish, cutting of WEDM.

Kravet [1] proposed a calculation method for a multi-cut finish-cutting process in WEDM. The number of machining operations was determined by minimizing the total machining time $\left(T_{\mathrm{m}}\right)=\Sigma_{1}^{n}$ (machining volume/ metal removal rate). In this expression, the machining volume and the metal removal rate are both functions of surface roughness, and they are difficult to estimate accurately. Furthermore, the approach to determine the optimal machining parameters for each operation such as pulse-on time, pulse-off time, etc. was not discussed.

Scott, Boyina and Rajurkar [2] used a factorial design method to determine the optimal combination of control parameters in WEDM, the measures of machining performance being the metal removal rate and the surface finish. It was found that the discharge current, the pulse duration and the pulse frequency are significant control factors. A total number of 729 experiments were conducted, and 32 machining-parameters settings that resulted in a better metal removal rate and surface roughness were determined by a numerical method. However, this approach requires too many experiments and finish cutting operations are not considered.

Tarng [3] applied a neural network with a simulated annealing (SA) algorithm to determine the optimal cutting parameters in WEDM. A neural network can easily formulate a model of the cutting parameters and performance by training and learning. This research focused on the rough cutting process only, where finish cutting number and offset value were not discussed. A multi-objective function, which is the weighted combination of surface roughness and machining speed, was adopted as the main objective function. However, this cannot provide the optimal machining parameters for an arbitrary desired surface roughness.

Machining-parameters setting tables, from rough to finish cutting, help the machine-user to make decisions

Table 1

Machining conditions

\begin{tabular}{ll}
\hline Item & Value \\
\hline Workpiece (anode) & SKD11 alloy steel \\
Electrode (cathode) & $\phi 0.25 \mathrm{~mm}$ brass wire \\
Workpiece height & $30 \mathrm{~mm}$ \\
Open voltage & $95 \mathrm{~V}$ \\
Servo reference voltage & $10 \mathrm{~V}$ \\
Fluid specific resistance & $1-3 \mathrm{~mA}$ \\
\hline
\end{tabular}

Table 2

Experimental design

\begin{tabular}{lccc}
\hline Control factor & Level 1 & Level 2 & Level 3 \\
\hline His & $\mathrm{a}$ & $\mathrm{b}$ & - \\
$T_{\text {on }}$ & 0.1 & 0.2 & 0.3 \\
$T_{\text {off }}$ & 3.2 & 8 & 12.8 \\
Feed & 2 & 5 & 8 \\
$D_{\text {ww }}$ & +5 & -20 & -45 \\
$P$ & 0.5 & 1.0 & 1.5 \\
\hline
\end{tabular}

Table 3

Machining conditions used in rough cutting

\begin{tabular}{lcccccl}
\hline Case & $T_{\text {on }}$ & $\mathrm{T}_{\text {off }}$ & Feed & $P$ & Wire tension & Wire speed \\
\hline $\mathrm{a}$ & 0.3 & 8 & 0.4 & 4 & 1200 & 5 \\
$\mathrm{~b}$ & 0.9 & 17.6 & 2.6 & 6 & 1000 & 9 \\
\hline
\end{tabular}


(a)

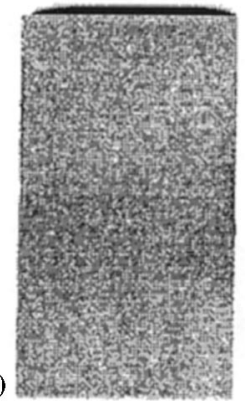

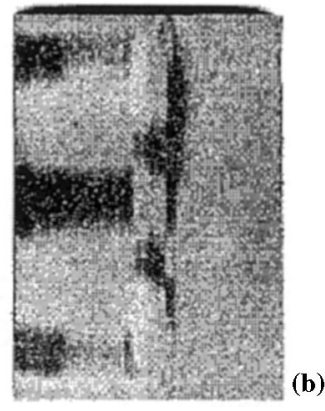

(b)

Fig. 2. The non-uniform area of a machined workpiece in the finish-cutting operation: (a) larger $D_{\text {ww }}$; (b) smaller $D_{\text {ww }}$.

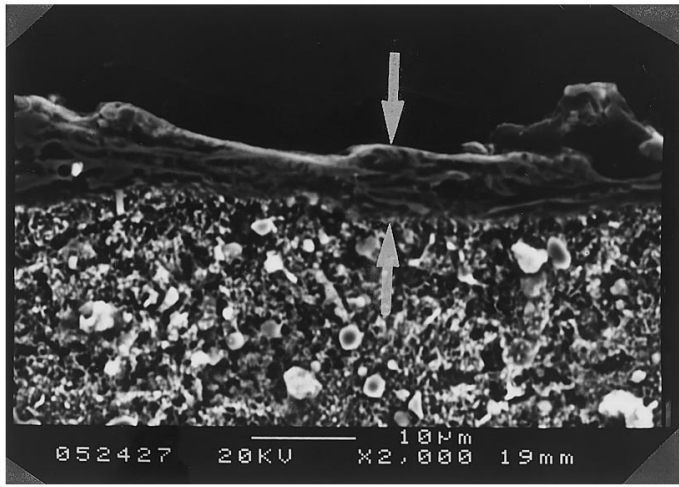

Fig. 3. Measurement of the white layer depth (SEM photograph).

Table 4

Computed $F$ value of $Y_{\text {gap }}, Y_{\text {ra }}$ and $Y_{\mathrm{s}}$

\begin{tabular}{lcccll}
\hline Factors & $Y_{\text {gap }}$ & $Y_{\text {ra }}$ & $Y_{\mathrm{d}}$ & $Y_{\mathrm{s}}$ & $F_{0.05, \mathrm{n} 1, \mathrm{n} 2}$ \\
\hline His & 0.177 & 0.002 & $4.031^{\wedge}$ & 0.300 & 5.99 \\
$T_{\text {on }}$ & $9.151^{*}$ & $47.403^{*}$ & $5.677^{*}$ & 1.705 & 5.14 \\
$T_{\text {off }}$ & 1.520 & 2.028 & 2.753 & 0.900 & 5.14 \\
Feed & 1.316 & $5.001^{\wedge}$ & 0.512 & 0.244 & 5.14 \\
$D_{\text {ww }}$ & $39.39^{*}$ & $10.476^{*}$ & $11.641^{*}$ & $5.696^{*}$ & 5.14 \\
$P$ & 0.187 & 2.576 & 0.213 & 0.163 & 5.14
\end{tabular}

* Significant factor.

$\wedge$ Sub-significant factor.

of the stages of machining operations, power-parameters settings, etc. These machining-parameters tables, based on the in-house experimentation of the manufacturer, are quite useful but are time-consuming to build. Moreover, this kind of machining decision is too conservative and does not lead to an optimal solution [2]. In recent years, some research has focused on the optimal determination of machining parameter in WEDM, but are still concerned mostly with the roughcutting process [2-4]. Even with several studies, optimal machining-parameters setting and process planning of WEDM still encounter some difficulties [5,6]. To be more specific, it is too costly and time-consuming; the performance is affected by too many parameters and the real mathematical models between machining parameters and performance are quite sophisticated and hard to derive. Hence, it is desirable to have a better understanding of the characteristics of finish-cutting process so that an effective approach to determine finish cutting parameters setting can be proposed. This can help machine-makers to establish optimal machining tables efficiently, and moreover, to achieve the goal of automatic process planning.

In this paper, the finish-cutting operation number and parameters setting of WEDM are the main concern. The influence of machining parameters (pulse-on time, table feed, flushing, distance between the wire periphery and the workpiece surface, and machining history (i.e. rough cutting conducted before finish cutting)) on the machining performance (gap width, surface roughness, white layer depth and finish-cutting area ratio) are analyzed first. The Taguchi quality design method and numerical analysis are used to find significant factors affecting the machining performance. Mathematical models relating the machining parameter and performance are established by regression, and non-linear programming using the feasible-direction algorithm is used to obtain the optimal machining parameters. Based on experimental data and numerical analysis, a practical strategy of multi-cut WEDM process planning from rough to finish cutting is proposed and verified.

\section{Experimental method}

\subsection{Experimental design}

Experiments were carried out on a Wire-EDM machine with an iso-energy pulse generator, developed by the Industrial Technology Research Institute (ITRI), Taiwan. The work material, electrode and other machining condition are given in Table 1.

According to the Taguchi quality design concept [7], a L18 mixed orthogonal arrays table was chosen for the experiments (Appendix A). Based on experience and related literature, six machining parameters: pulse-on time ( $\left.T_{\text {on }}\right)$; pulse-off time ( $\left.T_{\text {off }}\right)$; table feed (Feed); flushing pressure $(P)$; distance between wire periphery and workpiece surface $\left(D_{\mathrm{ww}}\right.$, Fig. 1$)$; and machining history (i.e. rough cutting conducted before finish cutting, (His)); were chosen for the controlling factors and each parameter was designed to have three levels denoted by 1, 2 and 3, as shown in Table 2. Levels 1 and 2 in Table 2 denoted by (a) and (b), respectively, are the rough cuts conducted in advance, their conditions are given in Table 3. The finish cutting results after the WEDMed process are evaluated in terms of the following measured machining performance: gap width $\left(Y_{\text {gap }}, \mu \mathrm{m}\right)$ surface roughness $\left(Y_{\mathrm{ra}}, \mu \mathrm{m}\right)$, white layer depth $\left(Y_{\mathrm{d}}, \mu \mathrm{m}\right)$ and finish-cutting area ratio $\left(Y_{\mathrm{s}}\right)$. The finish-cutting area ratio is defined as the real machining area divided by the whole area to be machined. It has a value 

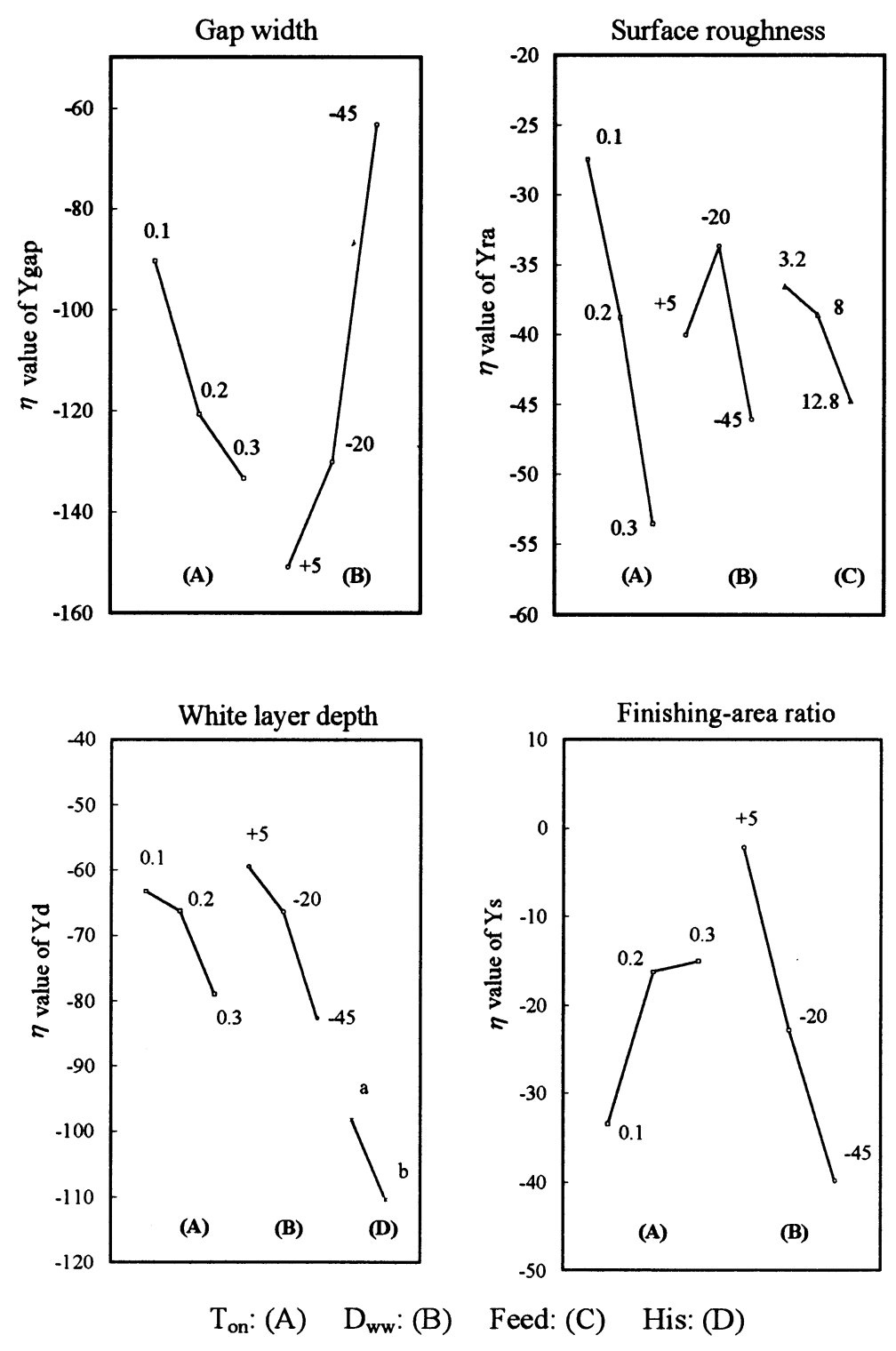

Fig. 4. The variation of $\eta$ values with machining parameters.

between 0 and 1 , the value 1 meaning that a whole area is machined. Owing to the non-uniform surface after the rough-cutting operation, some area may not be machined, as shown in the black area of Fig. 2, if a smaller $D_{\mathrm{ww}}$ is used. Hence, the ratio will be helpful to reflect the real finish cutting situation indirectly.

In Taguchi quality engineering [7], the characteristic that a higher value represents better machining performance, such as the finish-cutting area ratio, is called 'higher is better, HB'. Conversely, the characteristic that a lower value indicates better machining performance, such as the white layer depth, the gap width, and the surface roughness, is called lower is better, LB'. The signal-noise ratio, denoted by $S / N$ ratio, is a representation to find significant parameters from those controlling machining parameters by evaluation the minimum variance. For $\mathrm{HB}$ and $\mathrm{LB}$, the definition of the $S / N$ ratio for machining performance results $y_{\mathrm{i}}$ of $n$ repeated number (in this case $n=3, i=1,2,3$ ) are computed as:

HB: $S / N$ ratio $=1 /\left(\frac{1}{n}\left(\frac{1}{y_{1}^{2}}+\frac{1}{y_{2}^{2}}+\cdots+\frac{1}{y_{n}^{2}}\right)\right)$

LB: $S / N$ ratio $=1 /\left(\frac{1}{n}\left(y_{1}^{2}+y_{2}^{2}+\cdots+y_{n}^{2}\right)\right)$

Let $\eta=10 * \log (S / N$ ratio): then a larger $\eta$ (or larger $S / N$ ratio equivalently) is better for both $\mathrm{HB}$ and LB.

\subsection{Data measurements}

After being machined, the specimens were cleaned and their surface roughness $\left(Y_{\mathrm{ra}}\right)$ were measured using a Hommel T4000 Turbo-Roughness instrument. The cut- 


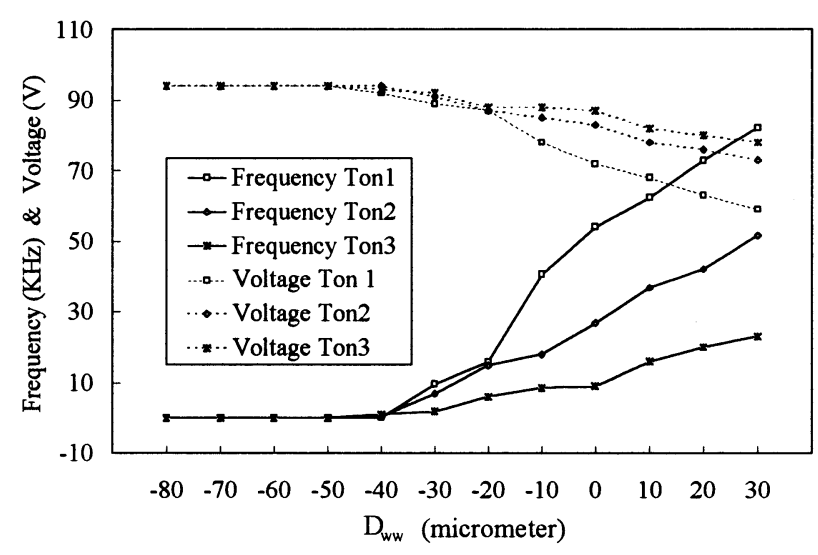

(a)

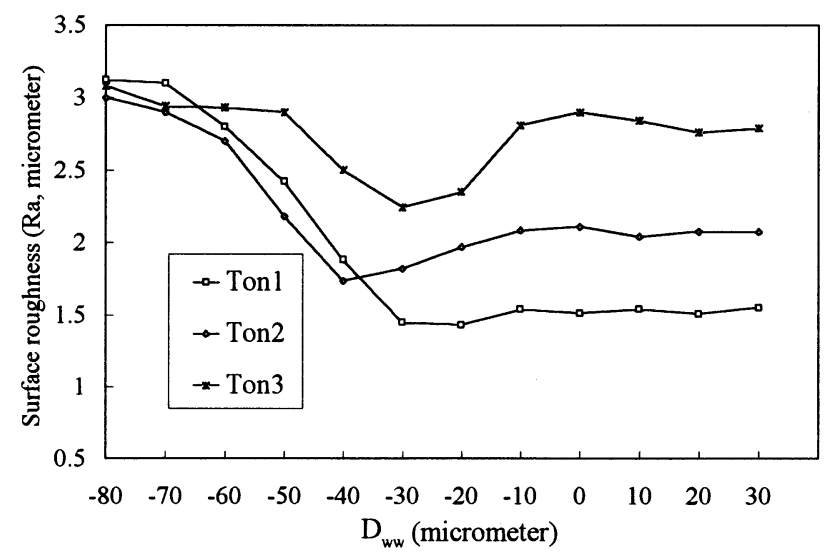

(b)

Fig. 5. (a) Sparking frequency and voltage vs $D_{\text {ww }}$; and (b) surface roughness vs $D_{\mathrm{ww}}$.

off value is $0.8 \mathrm{~mm}$ and the transverse length is $4.8 \mathrm{~mm}$. Gap width $\left(Y_{\text {gap }}\right)$ is measured by a short circuit system of the WEDM machine and a Nikon Measuring Micro- scope. As for the measurement of the white layer depth, specimens were sectioned transversely, mounted, ground, polished and etched. The etching reagent used was Fry's microetching solution $\left(5 \mathrm{~g} \mathrm{CuCl}_{2}, 40 \mathrm{ml} \mathrm{HCl}\right.$, $25 \mathrm{ml} \mathrm{C}_{2} \mathrm{H}_{5} \mathrm{OH}, 30 \mathrm{ml} \mathrm{H}_{2} \mathrm{O}$ ) and the etching time was about $10 \mathrm{~s}$. Then the specimens were photographed under a JSM-6300 scanning electron microscope (SEM), a typical microphotograph is shown in Fig. 3. The white layer depth is obtained by measuring the thickness of the top layer depicted between two arrows in Fig. 3. The finish-cutting area ratio $\left(Y_{\mathrm{s}}\right)$ was calculated approximately as the finish cutting area divided by the whole area to be cut under the microscope. All of the above values were obtained by taking the average of at least 15-20 measured data.

\section{The influence of finish-cutting parameters on machining performance}

The $\eta$ vales of machining parameters on performance are calculated (Appendix A). With analysis of variance (ANOVA) and the statistical $F$-test (Appendix B), it is found that $T_{\text {on }}$ and $D_{\mathrm{ww}}$ are the two dominant factors on the machining performance in the finish cutting process. Table 4 shows the computed $F$ values of six machining parameters on each performance. The sign ' $*$ ' indicates significant factors for the reason that the computed $F$-value is greater than $F_{0.05, \mathrm{n} 1, \mathrm{n} 2}$, the upper $95 \%$ point of the $F$-distribution having $n_{1}$ and $n_{2}$ degrees of freedom. Furthermore, the table feed-rate and the machining history also have an influence on the surface roughness and the white layer depth, respectively.

\subsection{Pulse-on time $\left(T_{\text {on }}\right)$}

Fig. 4 shows the variation of $\eta$ values with respect to

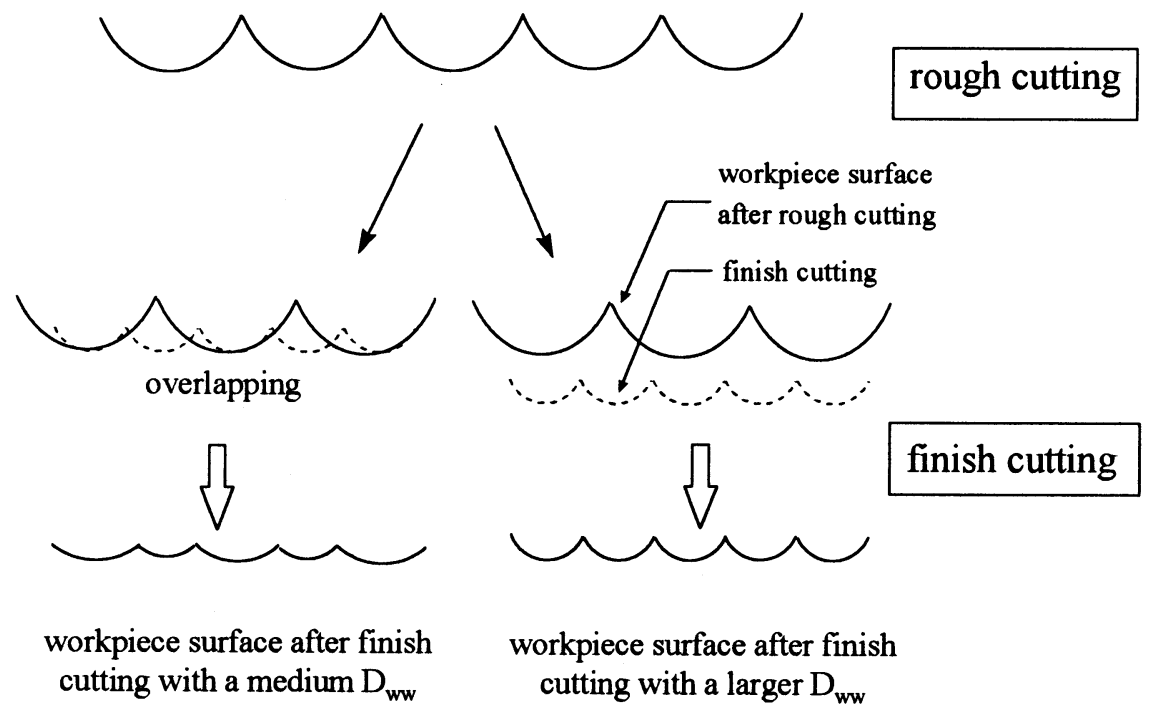

Fig. 6. Illustration of the surface generation mechanism in finish cutting. 
each machining parameter for machining performance of interest. It is found that larger $T_{\text {on }}$ results in larger $Y_{\text {gap }}, Y_{\text {ra }}, Y_{\mathrm{d}}$ and $Y_{\mathrm{s}}$ because larger pulse-on time means larger electrical discharging energy, which will produce a bigger crater. This is the same for the rough cutting process.

3.2. Distance between wire periphery and workpiece surface $\left(D_{w w}\right)$ :

In Fig. 4, a larger $D_{\text {ww }}$ results in larger $Y_{\text {gap }}$ and $Y_{\mathrm{s}}$, and smaller $Y_{\mathrm{d}}$ because the wire is closer to the workpiece. However, it causes $Y_{\text {ra }}$ to decrease first, then subsequently it is increased. In order to reveal the effect of $D_{\text {ww }}$ more comprehensively, some other experiments were conducted. Fig. 5(a, b) show the sparking frequency and average gap voltage, and the surface roughness, respectively, against $D_{\mathrm{ww}}$. It is reasonable that the sparking frequency is increased and the voltage is decreased with respect to the increase of $D_{\mathrm{ww}}$, as shown in Fig. 5(a). In Fig. 5(b), the varying trend of the $D_{\text {ww }}$ value on the surface roughness, mentioned previously, can be seen again. An idealistic finish-cutting mechanism is proposed in Fig. 6, which may explain the reason why a better $Y_{\text {ra }}$ can be obtained by using a negative and medium $D_{\mathrm{ww}}$ value (about $-30 \mu \mathrm{m}$ in this research). From Figs. 4 and 5, a negative and medium $D_{\text {ww }}$ can remove the surface peak of workpiece to minimize the surface roughness but the area will not be machined completely. There is no discharge for a smaller $D_{\text {ww }}$ because the distance between the workpiece surface and the wire periphery is larger than the minimum electrical discharging gap. Contrarily, the whole surface can be machined under a larger $D_{\mathrm{ww}}$, but it will take a longer time and result in a medium $Y_{\text {ra }}$. Hence, in order to obtain a smaller $Y_{\text {ra }}$, a negative and medium $D_{\mathrm{ww}}$ is appropriate. A larger $D_{\mathrm{ww}}$ should be used for a larger finish-cutting area ratio and smaller white layer depth.

Table 5

Computed machining-parameters setting in finish cutting

\begin{tabular}{lllllll}
\hline$T_{\text {on }}$ & Feed & $D_{\text {ww }}$ & $T_{\text {off }}$ & $F_{\mathrm{w}}$ & $V_{\mathrm{w}}$ & $\mathrm{P}$ \\
\hline 0.2 & 5.11 & 3 & 8 & 1200 & 3 & 1 \\
\hline
\end{tabular}

Table 6

Comparison of computed and experimental performance

\begin{tabular}{lccl}
\hline Performance & $Y_{\text {gap }}$ & $Y_{\text {ra }}$ & $Y_{\mathrm{d}}$ \\
\hline Computed & 18.3 & 1.97 & 2.8 \\
Experimental & 17.5 & 2.02 & 3.0 \\
Error (\%) & 4.41 & 2.54 & 7.14 \\
\hline
\end{tabular}

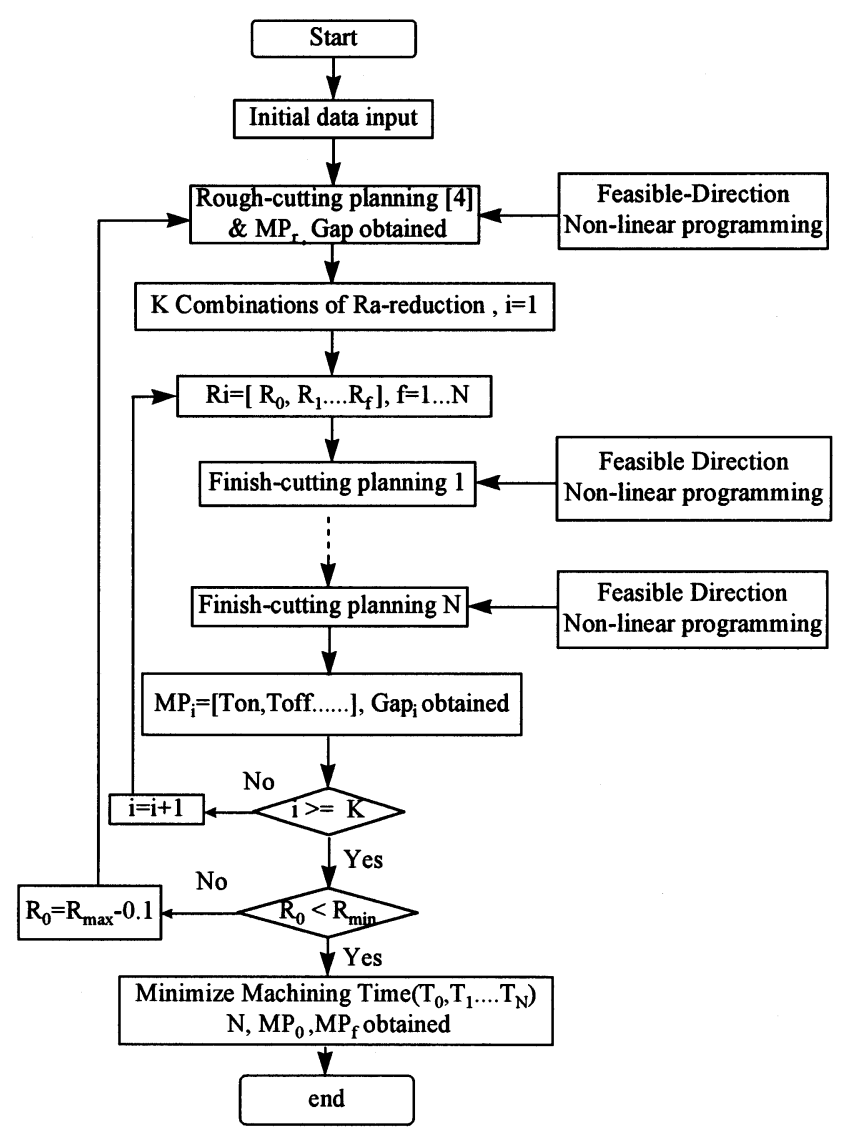

Fig. 7. Strategy of WEDM process planning.

In addition to $T_{\text {on }}$ and $D_{\mathrm{ww}}$, it is also found from Fig. 4 that a low table feed-rate can remove surface peaks effectively, and a rough cut with a high pulse electrical energy will result in a larger residual white layer depth after finish cutting.

\section{Machining planning}

In order to obtain specified dimensional accuracy and surface quality, and release the residual stress and heat deformation, multi-cut process planning from rough to finish cutting is necessary. The optimal machining parameters for each finish cutting operation can be obtained by seeking the maximum machining speed under the constraints of surface roughness, white layer depth and other constraints. A strategy is proposed and the feasible-direction non-linear programming method is adopted to solve this problem.

\subsection{Mathematical model of finish cutting}

Based on previous analysis, the pulse-on time, the distance between the wire periphery and the workpiece surface, and the table feed-rate were chosen as three 


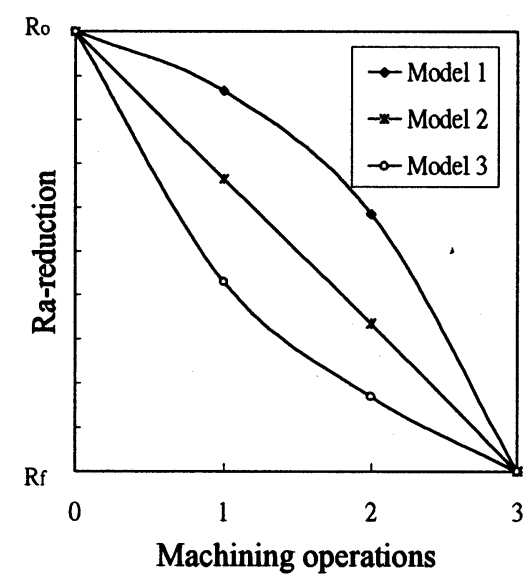

Model 1: $\quad Y=-0.25 e^{\frac{\ln \left(4 R_{0}-4 R_{f}+1\right)}{N} x}+\left(0.25+R_{0}\right)$

Model 2: $\quad \mathrm{Y}=\frac{\mathrm{R}_{\mathrm{f}}-\mathrm{R}_{0}}{\mathrm{~N}} \cdot \mathrm{X}+\mathrm{R}_{\mathbf{0}}$

Model 3: $\quad Y=-\ln \left[\frac{e^{R_{0}-R_{f}}-1}{N} \cdot X+1\right]+R_{0}$

Where

$\mathrm{N}: \quad$ finish-cutting number, $1 \ldots 4$

$X: 1$...N

$\mathrm{R}_{0}$ : surface roughness after rough-cutting

$\mathrm{R}_{\mathrm{f}}$ : desired final surface roughness

Fig. 8. Three models of surface roughness reduction in finish cutting.

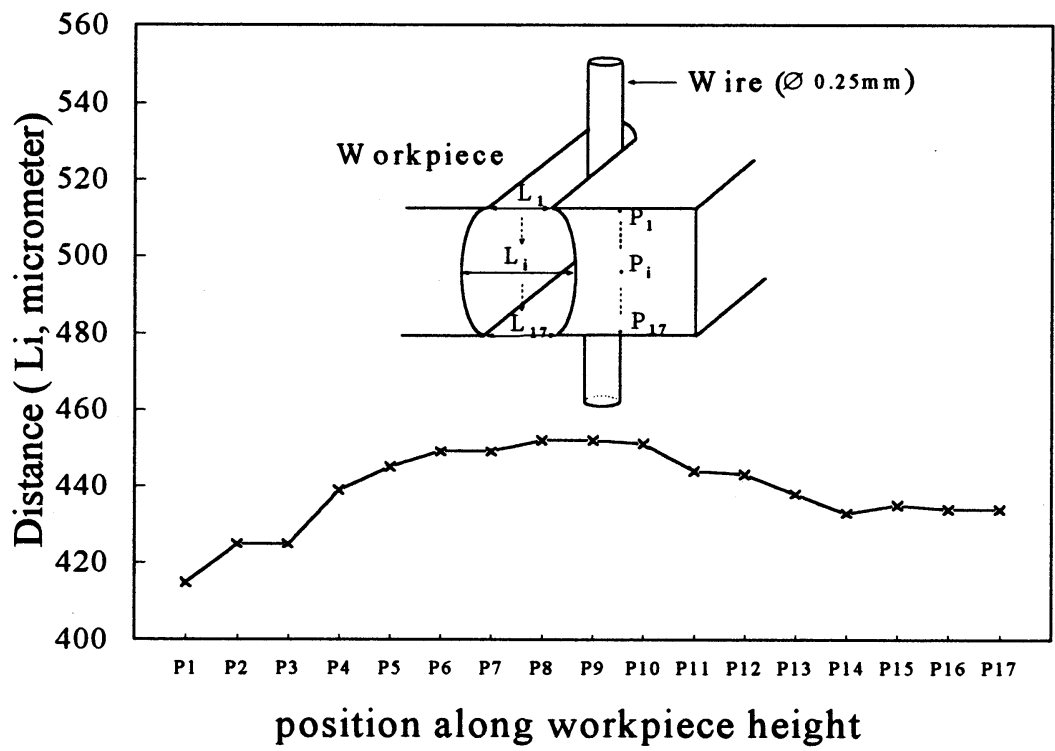

Fig. 9. Drum shape after rough cutting.

machining parameters that influence gap width, the surface roughness, and the white layer depth. It should be noted that the table feed-rate cannot be increased without constrain because retreat of the wire may occur, which will deteriorate the machined surface. The maximum table feed-rate is limited, since it should be compatible with the material removal ability. Hence, it is set as a function of $T_{\text {on }}$ and $D_{\text {ww }}$ : a larger $T_{\text {on }}$ and a smaller $D_{\text {ww }}$ will make a larger table feed-rate possible.

A mathematical model of machining parameters and performance is obtained by regression analysis, a general quadratic form as give below being chosen:

$Y=a+\sum_{i=1}^{p} a_{i} x_{i}+\sum_{i=1}^{p} \sum_{j=i}^{p} a_{i j} x_{i} x_{j}$

After numerical manipulations of the experimental data, the mathematical models are expressed in matrix form as follows:

$\mathbf{Y}=\mathbf{X A}$

$\mathbf{Y}=\left[Y_{\text {gap }} Y_{\text {sr }} Y_{\mathrm{d}} Y_{\text {max-feed }}\right]$

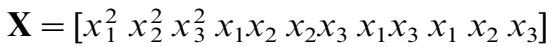

where,

$x_{1}=\log \left(T_{\text {on }}\right) / \max \left(\log \left(T_{\text {on }}\right)\right)$

$x_{2}=\log \left(R_{\mathrm{w}}-D_{\mathrm{ww}}\right) / \max \left(\log \left(R_{\mathrm{w}}-D_{\mathrm{ww}}\right)\right)$

$x_{3}=\log ($ Feed $) / \max (\log ($ Feed $))$

In this expression, $x_{1}$ is between 0 and $1, x_{2}$ is between 0.8813 and 1 , and $x_{3}$ is between 0.6505 and 1 , for this research. The matrix $\mathbf{A}$ is obtained as follows: 


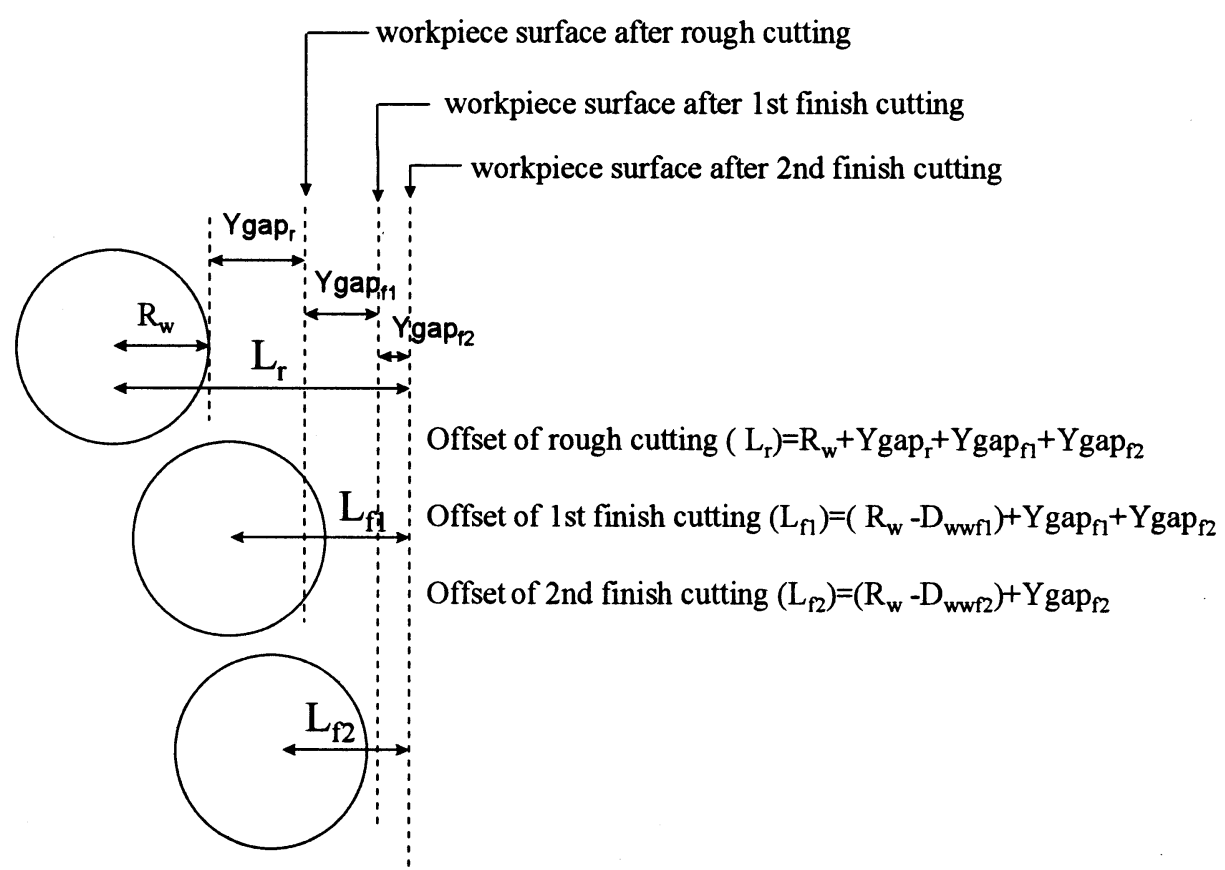

Table 7

Fig. 10. Illustration of offset value calculation.

Computed machining conditions

\begin{tabular}{llllllll}
\hline Process & $R_{\mathrm{a}}$ & $T_{\text {on }}$ & $T_{\text {off }}$ & Feed & $D_{\mathrm{ww}}$ & $V_{\mathrm{w}}$ & $F_{\mathrm{w}}$ \\
\hline Rough cutting & 3.2 & 1 & 17.6 & 2.4 & 258 & 9 & 1000 \\
First finish cutting & 2.03 & 0.2 & 8 & 3.94 & 148 & 3 & 1200 \\
Second finish cutting & 1.5 & 0.1 & 8 & 7.36 & 143 & 3 & 1 \\
\hline
\end{tabular}

$\mathbf{A}=\left[\begin{array}{cccc}0.2239 & 0.3112 & 0.3871 & 0.7624 \\ 31.0233 & 16.7417 & 55.0303 & -5.7811 \\ 0 & -0.9141 & 0 & 0 \\ -1.4086 & -2.5076 & -1.7875 & -6.7776 \\ 0 & 6.1140 & 0 & 0 \\ 0 & 0.3525 & 0 & 0 \\ 1.2920 & 2.1828 & 1.5384 & 6.0260 \\ -64.7591 & -35.1077 & -100.9884 & 20.7926 \\ 0 & -5.5826 & 0 & 0 \\ 33.7871 & 18.6696 & 46.7360 & -13.8933\end{array}\right]$

The correlation coefficients, [ $\left.R_{\text {gap }} R_{\mathrm{sr}} R_{\mathrm{d}} R_{\text {max-feed }}\right]=$ $[0.9821,0.9504,0.8326,0.9502]$, are computed using the following equation:

$$
R(x, y)=\frac{\sum(X-\bar{X})(Y-\bar{Y})}{\sqrt{\sum(X-\bar{X})^{2} \sum(Y-\bar{Y})^{2}}}
$$

Since all values are quite close to unity, it is concluded that the derived mathematical model is sufficient to represent the real machining performance. Based on these mathematical models, the gap width, the surface roughness, the white layer depth and the maximum feed-rate can be predicted correctly.
A general finish cutting problem can be formulated as follows:

Maximize: Feed-rate

Subject to:

$$
\begin{aligned}
& Y_{\mathrm{sr}} \leq R \\
& Y_{\mathrm{d}} \leq D \\
& x_{\mathrm{il}} \leq x_{\mathrm{i}} \leq x_{\mathrm{iu}} \\
& \text { (i=1,2,3; } x_{\mathrm{i} 1} \text { : lower bound; } x_{\mathrm{iu}} \text { : up- } \\
& \text { per bound) }
\end{aligned}
$$

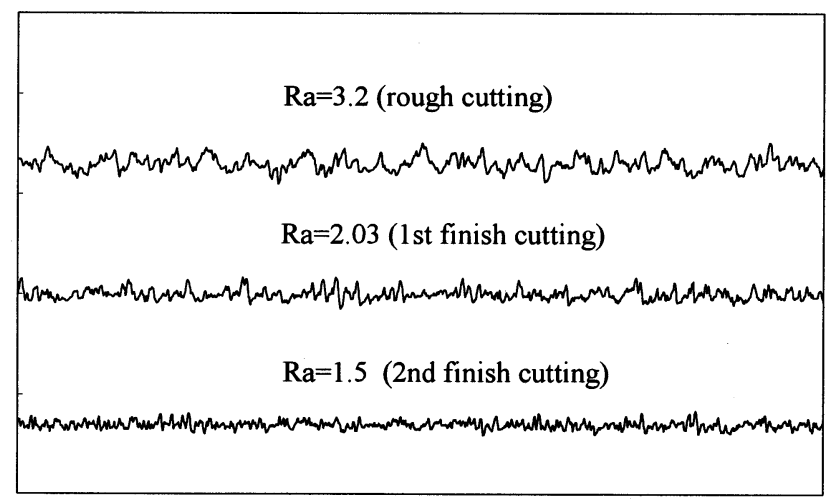

Fig. 11. Surface roughness profiles. 

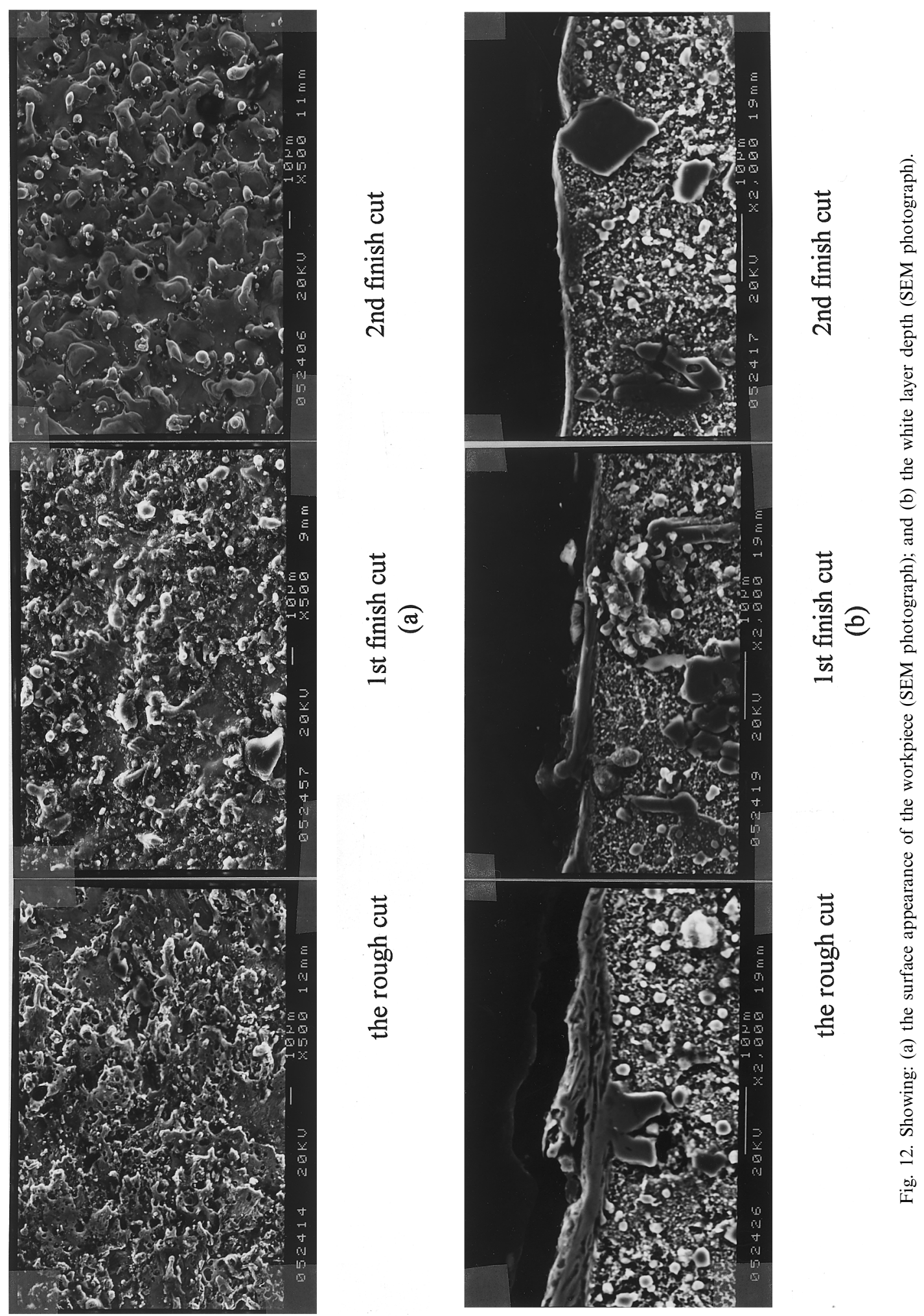


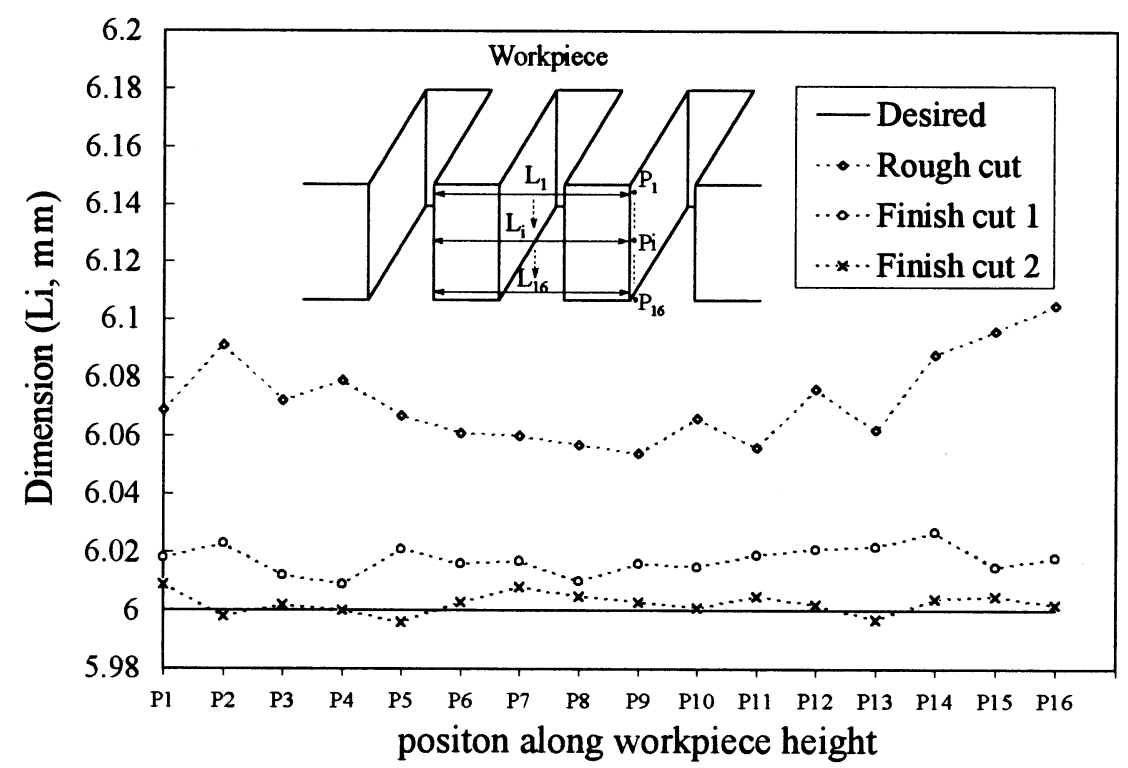

Fig. 13. Dimensional accuracy over the workpiece height.

$R$ and $D$ can be determined according to the desired surface roughness and white layer depth, respectively. By applying the feasible-direction method of non-linear programming with the above mathematical models $[4,8]$, the optimal machining parameters can be obtained. For illustration purposes, an example with $R=$ $2 \mu \mathrm{m}$ and $D=3 \mu \mathrm{m}$ resulted in the machiningparameters setting given in Table 5. After machining a workpiece using the suggested machining parameters, it is found that the computed and actual experimental machining performance is quite close, as indicated in Table 6, the error being less than $8 \%$ and thus acceptable.

\subsection{Rough and finish cutting planning}

In planetary die sinking EDM, Kishi [9] proposed three models for operation number planning and its associated machining-parameters determination: one for removing surface roughness only (corresponding to

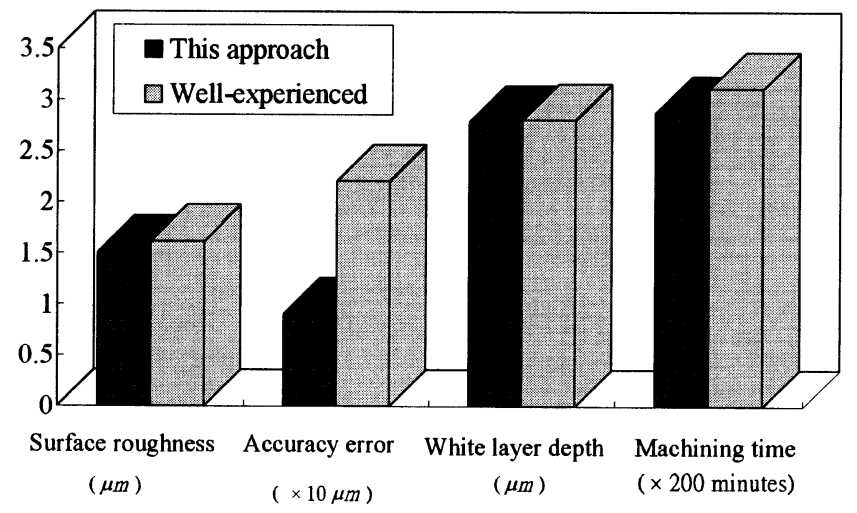

Fig. 14. Comparison of this approach with a well-skilled operation. a smaller $D_{\text {ww }}$ in this paper); and the others for minimizing the heat-effected zone (a larger $D_{\mathrm{ww}}$ in the present case). However, the three models were used numerically and independently in all cuts. In this paper, a strategy combining the removal of the heat-effected zone and surface roughness based on experiment is proposed and tested. A procedure of process planning to determine the machining operation number and parameters setting is shown in Fig. 7. Minimum machining time is the main objective and good surface quality and white layer depth are the constraints. To be more specific, this procedure is divided into five steps, as described below.

\subsubsection{Input initial data}

The desired final surface roughness $\left(R_{\mathrm{f}}\right)$ and machining length are specified and input.The initial surface roughness of rough cutting $\left(R_{0}\right)$ is set as $R_{\max }$.

\subsubsection{Rough-cutting process planning [4]}

With the goal of maximizing the metal removal rate and the constraints of $R<R_{0}$, the optimal machiningparameters setting of rough cut $M P_{\mathrm{r}}\left(T_{\text {on }}, T_{\text {off }}\right.$, Feed, $\left.V_{\mathrm{w}}, F_{\mathrm{w}}, P\right)$ is determined and the machining performance (gap width, surface roughness and machining time) is estimated by non-linear programming using the feasible-direction method.

\subsubsection{Surface roughness reduction algorithm}

There is no definite optimal strategy in determining the machining operation number. However, surface roughness is easy to measure and is used to represent the surface quality practically in WEDM. In order to determine an optimal operation number, three $R$ s-reduction models with different $\Delta R_{\mathrm{a}}$ (i.e. $R_{\mathrm{i}}-R_{\mathrm{i}-1}$ ), are proposed and evaluated. They are induced from: 
L18 table and $\eta$ values for surface roughness

\begin{tabular}{|c|c|c|c|c|c|c|c|c|c|c|c|}
\hline & \multicolumn{8}{|c|}{ Factors } & \multicolumn{2}{|c|}{$R_{\mathrm{a}}(\mu \mathrm{m})$} & \multirow{2}{*}{$\frac{\eta}{Y(\mathrm{db})}$} \\
\hline & His & $T_{\text {on }}$ & $T_{\text {off }}$ & Feed & $D_{\mathrm{ww}}$ & $P$ & e1 & $\mathrm{e} 2$ & 1 & 2 & \\
\hline 2 & 1 & 0.1 & 8.0 & 5 & -20 & 1.0 & 2 & 2 & 1.42 & 1.33 & -2.771 \\
\hline 3 & 1 & 0.1 & 12.8 & 8 & -45 & 1.5 & 3 & 3 & 2.41 & 2.49 & -7.784 \\
\hline 4 & 1 & 0.2 & 3.2 & 2 & -20 & 1.0 & 3 & 3 & 1.7 & 1.71 & -4.635 \\
\hline 7 & 1 & 0.3 & 3.2 & 5 & 5 & 1.5 & 2 & 3 & 2.95 & 2.8 & -9.176 \\
\hline 8 & 1 & 0.3 & 8.0 & 8 & -20 & 0.5 & 3 & 1 & 2.47 & 2.6 & -8.082 \\
\hline 9 & 1 & 0.3 & 12.8 & 2 & -45 & 1.0 & 1 & 2 & 2.98 & 2.88 & -9.339 \\
\hline 10 & 2 & 0.1 & 3.2 & 8 & -45 & 1.0 & 2 & 1 & 2 & 2.18 & -6.411 \\
\hline 11 & 2 & 0.1 & 8.0 & 2 & 5 & 1.5 & 3 & 2 & 1.47 & 1.44 & -3.258 \\
\hline 12 & 2 & 0.1 & 12.8 & 5 & -20 & 0.5 & 1 & 3 & 1.44 & 1.36 & -2.926 \\
\hline 17 & 2 & 0.3 & 8.0 & 2 & -45 & 0.5 & 2 & 3 & 2.61 & 2.73 & -8.532 \\
\hline 18 & 2 & 0.3 & 12.8 & 5 & 5 & 1.0 & 3 & 1 & 3.12 & 2.99 & -9.702 \\
\hline
\end{tabular}

Table 9

Total $\eta$ values of each level on surface roughness

\begin{tabular}{lllllrr}
\hline & His & $T_{\text {on }}$ & $T_{\text {off }}$ & Feed & $D_{\text {ww }}$ & $P$ \\
\hline 1 & -60.021 & -27.449 & -39.891 & -36.553 & -40.086 & -36.864 \\
2 & -59.868 & -38.787 & -38.282 & -38.586 & -33.724 & -40.034 \\
3 & & -53.651 & -42.716 & -44.749 & -46.078 & -42.990 \\
Sum & -119.888 & -119.888 & -119.888 & -119.888 & -119.888 & -119.888 \\
\hline
\end{tabular}

Model 1: $y=a \cdot \mathrm{e}^{b x}+c$

Model 2: $y=a \cdot x+b$

Model 3: $y=a \cdot \ln (b x)+c$

Model 2 contains constant $\Delta R_{\mathrm{a}}$ in the whole process. Model 1 has the character of larger $\Delta R_{\mathrm{a}}$ at the beginning and smaller $\Delta R_{\mathrm{a}}$ in the final operation, and Model 3 is the opposite of Model 1. These three surface roughness reduction models for a fixed number of finish cutting operations $(N)$, surface roughness after rough cut $\left(R_{0}\right)$ and desired final surface roughness $\left(R_{\mathrm{f}}\right)$, are shown in Fig. 8. Generally speaking, four finish cuts are sufficient, as found from the machining-parameters setting table of WEDM.

\subsubsection{Finish-cutting process planning}

In order to ensure that the components are free from any surface defects, the heat-effected material that should be removed in the finish cutting process is at least ten times the surface roughness vale (about $30 \mu \mathrm{m}$ in WEDM) [10]. Measurements in experiments also show that the deviation of the drum shape in the workpiece thickness direction (Fig. 9) is about 15-25 $\mu \mathrm{m}$. In other words, a total of about $50 \mu \mathrm{m}$ of material should be removed in the finish-cutting operation. In this strategy, the first finish cut is aimed to minimize the heat-effected zone and the drum shape caused by rough cutting whilst satisfying the desired surface roughness. From several experiments, it is found that the use of $D_{\text {ww }} \geq 15 \mu \mathrm{m}$ can achieve this goal. The white layer after rough cutting should be removed in finish cutting. Since its depth is about $7-10 \mu \mathrm{m}$, the white layer depth constraint $(D)$ is chosen to be equal to or smaller than $3 \mu \mathrm{m}$ in this research. All of the surface roughness reduction combinations of the three models have been taken into consideration.

\subsubsection{Optimal solution}

For each surface roughness reduction model, the total machining time can be summarized as follows:

$$
\begin{aligned}
T= & \left(\frac{\text { length }}{\text { feed }}\right) \text { rough }- \text { cutting }+\sum_{j=1}^{N}\left(\frac{\text { length }}{\text { feed }}\right) \text { finish } \\
& - \text { cutting }
\end{aligned}
$$

By seeking the minimum machining time, the optimal operation number and surface roughness reduction 
model can be obtained. At the same time, the machining parameters of rough and finish cuts are determined, and the performance (gap width, machining time mainly) is predicted. Each offset value for each operation is calculated, as shown in Fig. 10.

After checking many cases, it is found that a roughcutting process planning under maximum metal removal rate and a finish-cutting process planning using surface roughness reduction Model 3 can achieve the minimum machining time.

\subsection{Illustrated example}

To verify the proposed strategy, an example of process planning from rough to finish cutting is selected. The cutting length is $500 \mathrm{~mm}$ and the desired surface roughness is $R_{\mathrm{a}}=1.5 \mu \mathrm{m}$. The computed output show that one rough cutting and two finish cuttings are required, and optimal machining-parameters setting are given in Table 7. The process planning results are reasonable, since similar process planning is found in the machining-parameters setting tables of commercially available machines. A workpiece was machined using the machining parameters of Table 7. Surface roughness profiles for the machined surface in different cuts were measured and are drawn in Fig. 11. It is found that the desired surface roughness can be obtained in the last finish cutting. The workpiece surface appearance after a rough cutting and finish cuttings are given in Fig. 12(a). The rough cutting results in deeper craters and evident molten metal, whilst finish cutting results in a shallow crater and a low density of appendages and pockmarks. From Fig. 12(b), it is also found that the final finish cutting results in a thin and straight white layer of thickness less than $3 \mu \mathrm{m}$. Fig. 13 shows that the dimensional accuracy over the workpiece height is within $10 \mu \mathrm{m}$ in the final finish cut. Comparison of the proposed approach with that of a well-skilled operator is shown in Fig. 14. It is obvious that the proposed approach can achieve a better surface quality. It takes less machining time and, in particular, a more accurate value of the average dimension over the workpiece height can be obtained.

\section{Conclusions}

An attempt has been made to determine the number of machining operations and machining parameters efficiently in WEDM. Qualitatively, the pulse-on time $\left(T_{\text {on }}\right)$ and the distance between the wire periphery and the workpiece surface $\left(D_{\mathrm{ww}}\right)$ have been found to be significant factors in finish cutting performance (gap width, surface roughness, finish-cutting area ratio). It has also been found that a medium $\mathrm{D}_{\mathrm{ww}}$ (about -30 $\mu \mathrm{m}$ in this research) can achieve a better surface roughness, but the whole surface will not be machined. In other words, adjusting $T_{\text {on }}$ and $D_{\text {ww }}$ can control the finish cutting process.

Applying the feasible-direction non-linear programming method, optimal machining parameters can be obtained. Experimental results show that the approximate mathematical models can predict the machining performance within an acceptable error. Moreover, a strategy to determine the finish-cutting operation numbers and the parameters setting has been proposed and verified. It is concluded that rough-cutting process planning under maximum metal removal rate and finish cutting process planning using Model 3 of surface roughness reduction, can achieve minimum machining time. The proposed approach, compared with that of a well-skilled operator, can achieve a better surface quality and take less machining time and, in particular, accurate dimensional accuracy can be obtained.

\section{Nomenclature}

$R_{\mathrm{w}} \quad$ wire radius

Feed table feed-rate $\left(\mathrm{mm} \mathrm{min}^{-1}\right)$

$T_{\text {on }} \quad$ pulse-on time $(\mu \mathrm{s})$

$T_{\text {off }} \quad$ pulse-off time $(\mu \mathrm{s})$

$P \quad$ flushing pressure $\left(\mathrm{kg} \mathrm{cm}^{-2}\right)$

$V_{\mathrm{w}} \quad$ wire speed $\left(\mathrm{m} \mathrm{min}^{-1}\right)$

$F_{\mathrm{w}} \quad$ wire tension $(\mathrm{gf})$

$D_{\mathrm{ww}}$ distance between the wire periphery and the workpiece surface in finish cutting $(\mu \mathrm{m})$ (Fig. 1)

His machining history (rough-cutting conducted before finish cutting)

$Y_{\text {gap }}$ removal gap width $(\mu \mathrm{m})$

$Y_{\text {ra }}$ surface roughness $(\mu \mathrm{m})$

$Y_{\mathrm{d}} \quad$ white layer depth $(\mu \mathrm{m})$

$Y \mathrm{~s} \quad$ finish-cutting area ratio $(\mu \mathrm{m})$

\section{Appendix A}

A L18 mixed orthogonal arrays table and the computed $\eta$ values of surface roughness are given in Table 8. Taking $T_{\text {on }}$ on $Y_{\text {ra }}$ as an example, the $\eta$ values of three levels can be summarized as follows:

Level 1: $\eta_{\text {on } 1}=-(4.299+2.771+7.784+6.400+$ $3.258+2.926)=-27.449$

Level $2: \quad \eta_{\text {on } 2}=-(4.635+7.461+6.474+6.550+$ $7.177+6.490)=-38.787$

Level 3: $\eta_{\text {on3 }}=-(9.176+8.082+9.339+8.820+$ $8.532+9.702)=-53.651$ 
Similarly, $\eta$ values for the other parameters on other machining performance can be evaluated. Table 9 shows the total $\eta$ values of the levels of six parameters on surface roughness.

\section{Appendix B. Analysis of variance and F-test}

$S_{m}=\frac{\left(\sum \eta i\right)^{2}}{18}, S_{T}=\sum \eta i^{2}-S_{m}$

$S_{A}=\frac{\sum \eta A i^{2}}{N}-S_{m}, S_{E}=S_{T}-\sum S_{A}$

$V_{A}=\frac{S_{A}}{f_{A}}, F_{A 0}=\frac{V_{A}}{V_{E}}$

where $S_{\mathrm{T}}$ is the sum of squares due to total variation; $S_{\mathrm{m}}$ is the sum of squares due to mean; $S_{\mathrm{A}}$ is the sum of squares due to factor $A$ ( $A=$ Feed, $T_{\text {on }}, T_{\text {off }}$, His, $D_{\mathrm{ww}}$, and $P$ ); $S_{\mathrm{E}}$ is the sum of squares due to error; $\eta_{i}$ is the $\eta$ value of each experiment $(i=1-18) ; \eta_{\mathrm{Ai}}$ is the sum of $i$ level of factor $A(i=1,2$ or $\mathrm{i}=1,2,3) ; N$ is the repeating number of each level of factor $A ; f_{\mathrm{A}}$ is the degree of freedom of factor $A ; V_{\mathrm{A}}$ is the variance of factor $A$; and $F_{\text {Ao }}$ is the $F$-test value of factor $A$.

\section{References}

[1] A.T. Krvaets, Planning of the wire-EDM process, in: Proceedings of the 10th International Symposium For Electro-Machining, (ISEM-10), Magdeburg, Germany, 1992, pp. 218-222.

[2] D. Scott, S. Boyina, K.P. Rajurkar, Analysis and optimization of parameter combination in wire electrical discharge machining, Int. J. Prod. Res. 29 (11) (1991) 2189-2207.

[3] Y.S. Tarng, S.C. Ma, L.K. Chung, Determination of optimal cutting parameters in wire electrical discharge machining, Int. J. Mach. Tools Manuf. 35 (12) (1995) 1693-1701.

[4] Y.S. Liao, J.T. Huang, H.C. Su, A study on the machiningparameters optimization of the wire electrical discharge machining, J. Mater. Process. Technol. 71 (1997) 487-493.

[5] M.S. Phadke, Quality Engineering Using Robust Design, Prentice-Hall, Eaglewood, Cliffs, NJ, 1989.

[6] J. Matsuda, S. Tomishige, K. Tanaka, H. Yano, Evaluation of performance of WEDM by parameter design, Proceedings of the 9th International Symposium For Electro-Machining, (ISEM-9), Nagoya, Japan, 1989, pp. 68-71.

[7] P.J. Ross, Taguchi Techniques For Quality Engineering, McGraw-Hill, New York, 1989.

[8] G.N. Vanderplaats, Numerical Optimization Techniques for Engineering Design: with Application, McGraw-Hill, New York, 1984.

[9] M. Kishi, S. Suzuki, S. Araya, Optimization of multi-stage planetary machining parameters in NC die sinking EDM, Proceedings of the 9th International Symposium For Electro-Machining, (ISEM-9), Nagoya, Japan, 1989, pp. 34-37.

[10] L.C. Lee, L.C. Lim, V. Narayanan, V.C. Venkatesh, Quantification of surface damage of tool steels after EDM, Int. J. Mach. Tools Manuf. 28 (4) (1988) 359-372. 